\title{
Balancing Charge Carrier Transport in a Quantum Dot P-N Junction toward Hysteresis-Free High-Performance Solar Cells
}

Yuljae Cho, ${ }^{\dagger, \nabla_{\odot}}$ Bo Hou, ${ }^{\dagger, \nabla_{\odot}}$ Jongchul Lim, ${ }^{\dagger}$ Sanghyo Lee, ${ }^{\dagger}$ Sangyeon Pak, ${ }^{\dagger}$ John Hong, ${ }^{\dagger \odot}$ Paul Giraud, ${ }^{\dagger}$ A-Rang Jang, ${ }^{\dagger}$ Young-Woo Lee, ${ }^{\dagger, \S \odot ~ J u w o n ~ L e e, ~}{ }^{\dagger}$ Jae Eun Jang, ${ }^{\| \odot}$ Henry J. Snaith, ${ }^{\ddagger \odot}$

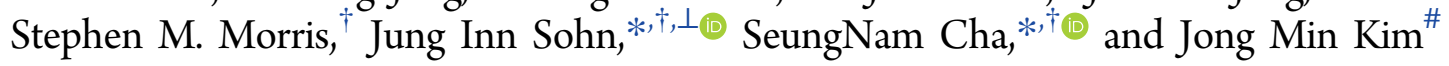

${ }^{\dagger}$ Department of Engineering Science, University of Oxford, Parks Road, Oxford OX1 3PJ, United Kingdom

${ }^{\ddagger}$ Department of Physics, Clarendon Laboratory, University of Oxford, Parks Road, Oxford OX1 3PU, United Kingdom

${ }^{\S}$ Department of Energy Systems, Soonchunhyang University, Asan, Chungcheongnamdo 31538, Republic of Korea

"Department of Information and Communication Engineering, Daegu Gyeongbuk Institute of Science and Technology, Daegu 711-873, Republic of Korea

${ }^{\perp}$ Division of Physics and Semiconductor Science, Dongguk University, Seoul 100-715, Republic of Korea

\#Department of Engineering, University of Cambridge, 9 JJ Thomson Avenue, Cambridge CB3 0FA, United Kingdom

\section{Supporting Information}

ABSTRACT: In a quantum dot solar cell (QDSC) that has an inverted structure, the QD layers form two different junctions between the electron transport layer (ETL) and the other semiconducting QD layer. Recent work on an inverted-structure QDSC has revealed that the junction between the QD layers is the dominant junction, rather than the junction between the ETL and the QD layers, which is in contrast to the conventional wisdom. However, to date, there have been a lack of systematic studies on the role and importance of the QD heterojunction structure on the behavior of the solar cell and the resulting device performance. In this study, we have systematically controlled the structure of the QD junction to balance charge transport, which demonstrates that the position of the junction has a significant effect on the hysteresis effect, fill factor, and solar cell performance and is attributed to balanced charge transport.

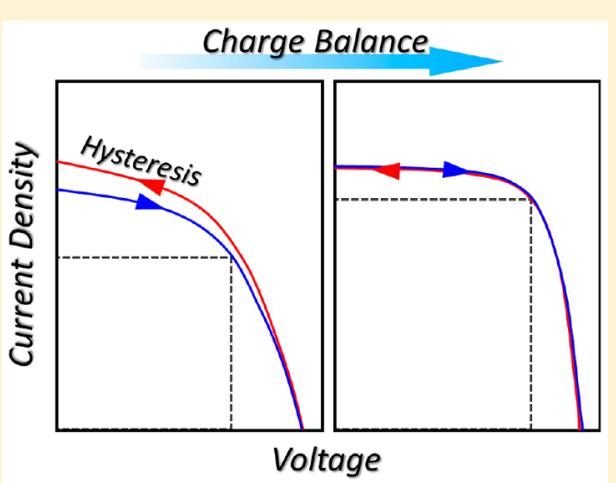

$\mathrm{H}$ arvesting photon energy has attracted tremendous interest because it is an environmentally friendly solution to the global energy demands in response to the exhaustion of fossil fuels. ${ }^{1,2}$ In accordance with the development of advanced materials for photon energy harvesting, the field of solar cells has continued to make steady technological progress. ${ }^{3}$ In particular, colloidal quantum dots (CQDs) have been shown to be one of the more promising materials for solar cell technology due to the facile and costeffective synthesis process as well as the extensive tunability of the bandgap. ${ }^{4-6}$ Among the various CQDs that have been developed, lead-based QDs such as lead sulfide ( $\mathrm{PbS}$ ) have been considered as the most attractive CQDs for photon harvesting devices because they exhibit a large Bohr radius ( $a=$ $20 \mathrm{~nm}$ ), wide bandgap tuning range $\left(E_{\mathrm{g}}=0.4-3.0 \mathrm{eV}\right)$, and large light absorption coefficients. ${ }^{6-8}$ Consequently, rapid progress in the area of $\mathrm{PbS}$ quantum dots solar cells (QDSCs) has been made over the past decade. ${ }^{5-8}$
For a PbS QDSC, the most common architecture consists of the following arrangement: electron transport layer (ETL)/ntype QD layers/p-type QD layers. ${ }^{9-18}$ Among the various strategies that have been developed to improve the performance of QDSCs, tailoring of the junction interfaces between an ETL and the PbS QD layers, as well as between the n-type and p-type QD layers, has been found to be crucial for highperformance QDSCs. ${ }^{9-18}$ However, there are different viewpoints in the literature about which junction plays the dominant role in the efficient transport and collection of the photogenerated charges. A very recent study has revealed that the junction formed between the $\mathrm{n}$ - and p-type QD layers is the dominant junction rather than the junction between the ETL and QD layers as was previously believed. ${ }^{19}$ Despite the recent finding, there is still a lack of understanding of the behavior of

Received: January 26, 2018

Accepted: March 28, 2018

Published: April 3, 2018 

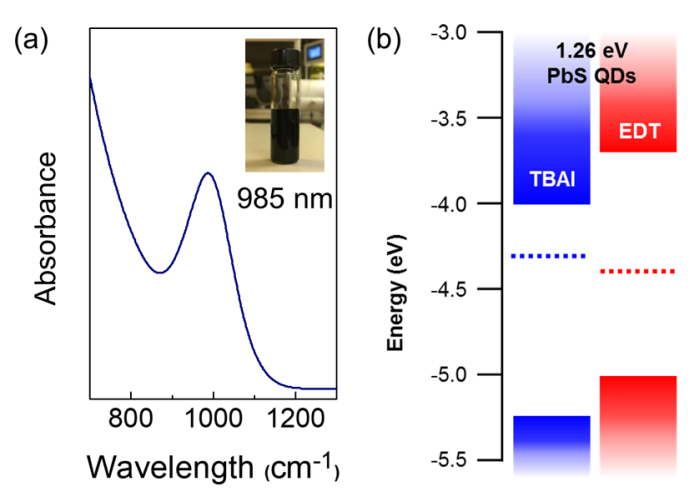
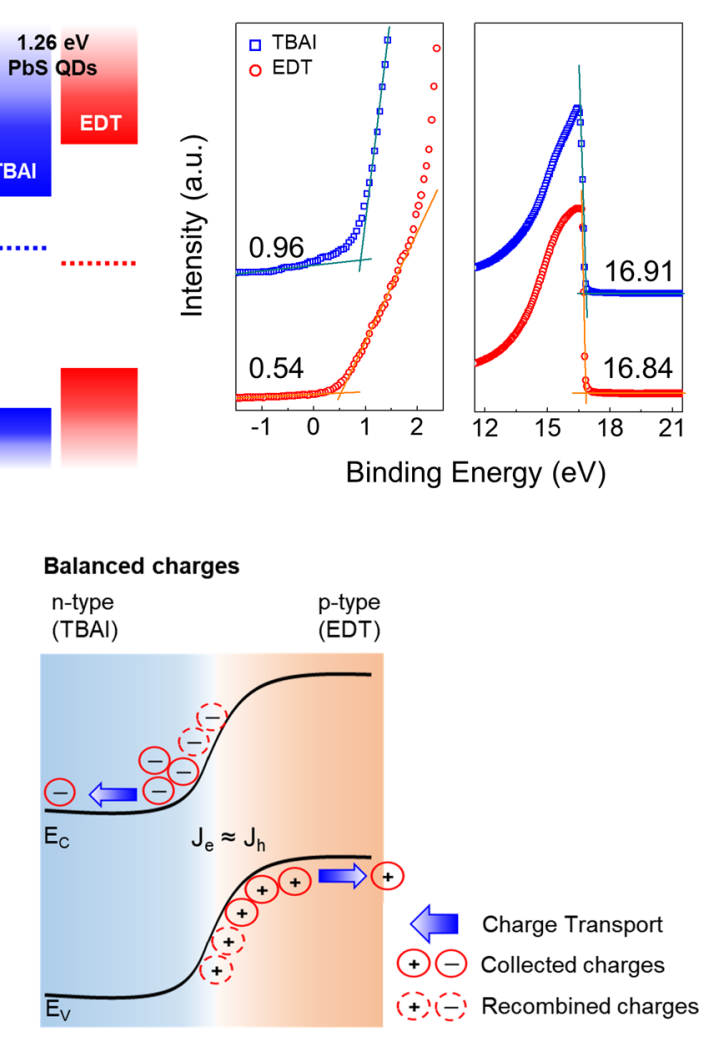

(c)

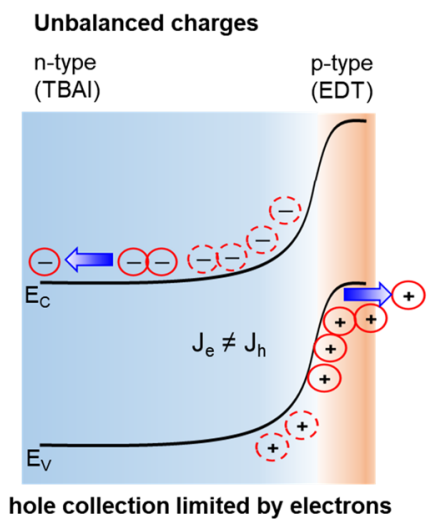

Figure 1. (a) Absorption spectrum of the as-synthesized PbS QDs measured using UV-vis spectroscopy (inset: photograph of the QDs in a solution of toluene). (b) Energy levels of the TBAI- and EDT-treated QD films (left diagram) and results obtained from UPS measurements (right plots). (c) Two-dimensional illustration of the charge carrier balance scheme in a typical $\mathbf{p}-\mathrm{n}$ junction structured solar cell when the lifetime and mobility of the charge carriers in the n-type layers and p-type layers are equivalent. $J_{\mathrm{e}}$ and $J_{\mathrm{h}}$ denote the electron and hole current densities, respectively.

the charge carriers in accordance with the QD junction architecture. Therefore, further investigation of the role of the $\mathrm{QD} \mathrm{p}-\mathrm{n}$ junction from a device operation point of view is highly desirable because the QD junction directly influences the behavior of the photogenerated charge transport, the formation of detrimental space charges, hysteresis characteristics, and ultimately the QDSC performance.

In this Letter, we have systematically controlled the structure of the QD $\mathrm{p}-\mathrm{n}$ junction in $\mathrm{PbS}$ QDSCs by manipulating the ratio of the number of n-type QD (tetrabutylammonium iodide (TBAI)) layers to the number of p-type (1,2-ethanedithiol (EDT)) QD layers so as to investigate the correlation between the location of the junction and the corresponding charge transport behavior. Toward this end, the photoelectronic and electrical properties of $n$ - and p-type QD films are analyzed to better understand the behavior of the charge transport in a QD junction, which provides a design motif for the ideal junction structure. Following analysis of the characteristics of the QD films, we demonstrate that the junction structure has a significant effect on the resulting solar cell performance, such as the fill factor (FF) and power conversion efficiency (PCE), which is attributed to balanced charge transport from the QD layers to the electrodes. By controlling the optimal charge balancing point through structural modification to the QD junction, a higher FF (greater than 70\%), reduced hysteresis, and consequently a significantly enhanced PCE ( 9\%) are achieved. We believe that this enhancement in efficiency is due to a balance of the charges at the QD $\mathrm{p}-\mathrm{n}$ junction, which suppresses the formation of space charges that can arise through an imbalance in the charge carrier transport. ${ }^{20,21}$ In contrast, we observe severe hysteresis, a low FF, and consequently a decrease in the PCE in QDSCs when the charge transport is not balanced, which occurs due to the formation of space charges and high series resistances. ${ }^{20,21}$ In addition, we demonstrate a significant improvement in power generation when comparing two types of solar modules that consist of a series of QDSCs that are either charge-imbalanced or -balanced, and we attribute the improved power generation to improved characteristics regarding the internal resistances (low $R_{\mathrm{s}}$ and high $R_{\mathrm{sh}}$ ) for the latter type of solar cells.

For fabrication of the PbS QDSCs, PbS QDs were first synthesized and purified according to the processes described in our previous work. ${ }^{6,8}$ Further details of the fabrication process and thickness of each layer are provided in the Methods section. Figure S1 shows a schematic of a QDSC structure, where the labels TBAI and EDT indicate the different types of ligands that were used in the $\mathrm{PbS} \mathrm{QD}$ layers; these have been the most frequently employed ligands as they exhibit good stability and high PCE among the types of QD ligands that have been studied to date. ${ }^{9-14}$ The as-prepared colloidal PbS QDs in toluene at a concentration of $50 \mathrm{mg} \mathrm{mL}^{-1}$ are shown in the inset of Figure 1a, and it was found that the bandgap was $1.26 \mathrm{eV}$ from measurements of the absorption spectrum of the QD solution using an ultraviolet-visible spectrometer (UVvis), as shown in Figure 1a. A solid-state ligand exchange process was applied in order to substitute the insulating oleic acid (OA) ligands that were initially attached to the PbS QDs for the semiconducting TBAI and EDT ligands. Fourier 
(a)

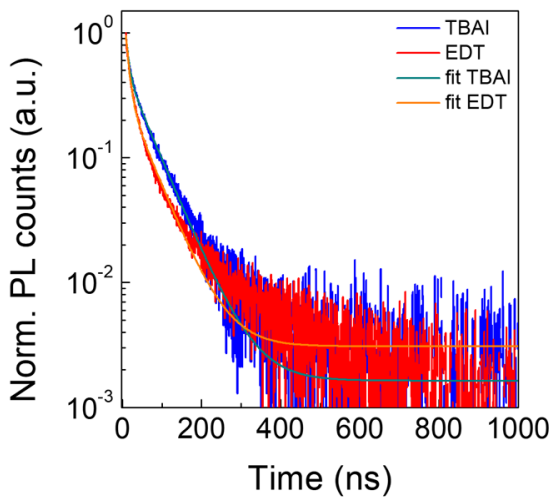

(c)

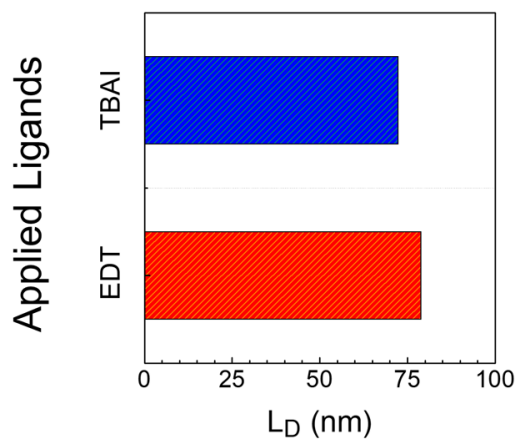

(b)
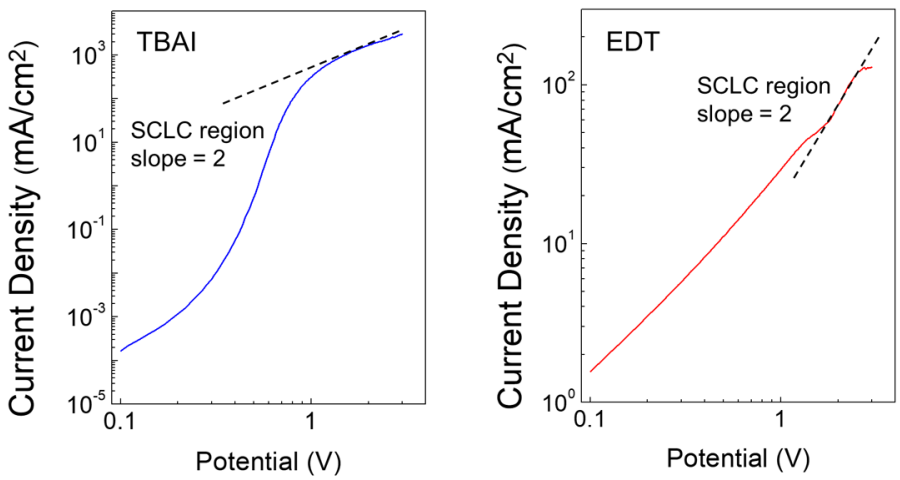

(d)
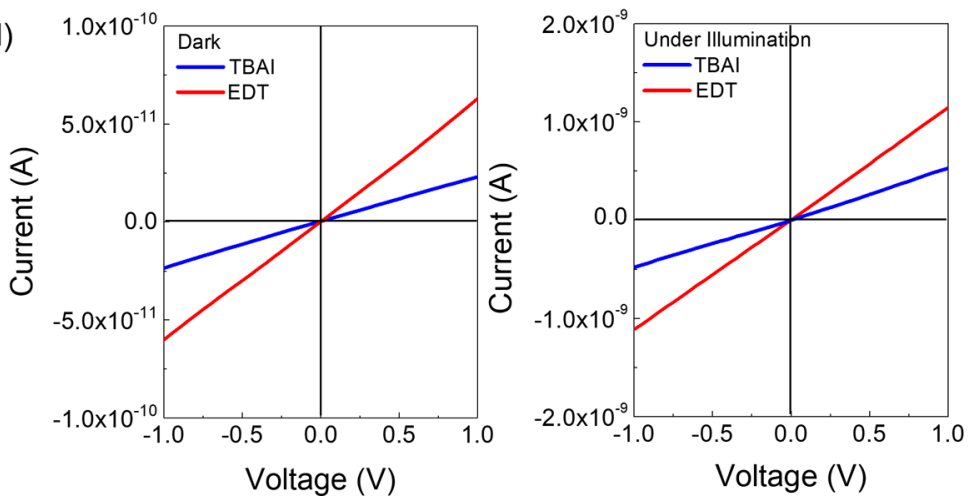

Figure 2. (a) Time-resolved photoluminescence of the TBAI- and EDT-passivated PbS QDs films. (b) Current-voltage curves for an electrononly (TBAI) (left plot) and a hole-only (EDT) (right plot) device. The mobility of each layer was extracted from the SCLC region $($ slope $=2$ ). (c) Calculated diffusion lengths of the charge carriers in the QD film treated with either TBAI (blue) or EDT ligands (red). (d) Conductivity measurements of the TBAI- (blue) and EDT-treated (red) films carried out in dark conditions and under illumination (white light).

transform infrared spectroscopy (FTIR) measurements revealed a noticeable decrease in the vibration peaks of the $-\mathrm{CH}_{2}$ and $-\mathrm{COO}$ bonds, as shown in Figure S2, indicating the successful removal of the OA from both types of QD films using a solid-state ligand exchange scheme. ${ }^{15}$

Following this, the energy bands for TBAI- and EDT-treated QD films were measured using ultraviolet photoelectron spectroscopy (UPS). As shown in Figure 1b, the TBAI- and EDT-treated QD films were identified as being n-type and ptype, respectively, which originates from the shift of the bandedge by electrostatic forces that are present in the treated ligands. ${ }^{9,22}$ Therefore, when EDT-treated QDs are deposited onto TBAI-treated QDs, a QD $\mathrm{p}-\mathrm{n}$ junction is formed between the separate QD layers. In a typical QDSC structure (Figure S1), two junctions are formed between (i) the zinc oxide ( $\mathrm{ZnO})$ ETL and the n-type QD layer and (ii) the n-type and ptype QD layers. ${ }^{9,11,19}$ However, we have considered the latter junction (the QD $\mathrm{p}-\mathrm{n}$ junction) in more detail in an effort to balance the charge carrier transport because it is the dominant junction in the structure and, moreover, the $\mathrm{ZnO}$ layer $(50 \mathrm{~nm})$ is considerably thinner than both the n-type and p-type QD layers (total $300 \mathrm{~nm}$ ). ${ }^{19}$ Figure $1 \mathrm{c}$ depicts the charge transport behavior in a $Q D p-n$ junction when the diffusion length of the charge carriers in the $\mathrm{n}$ - and $\mathrm{p}$-type materials are similar to one another (which will be discussed in more detail shortly). As shown in Figure 1c, left, when either the n- or p-type layer in the junction is larger than the other layer, the collection of electrons and holes becomes unbalanced due to differences in the lengths of travel for the charge carriers when they have similar diffusion lengths. As a result, the imbalance in charges results in space charges being formed across the junction, which leads to less efficient charge transport and severe hysteresis in the device characteristics. In contrast, in a charge-balanced system, through structural modification of the QD $p-n$ junction (Figure 1c, right), the formation of space charges is suppressed and thus charge transport and collection are more efficient than a charge-imbalance architecture. Consequently, a significantly enhanced FF and negligible hysteresis in the QDSC are observed, which lead to a concomitant improvement in the solar cell performance. ${ }^{23-25}$

In order to confirm these postulates, we first characterized the photoelectronic and electrical properties of the TBAI- and EDT-treated QD layers as the charge carrier transport in a junction-structured solar cell primarily depends on the diffusion length of each photogenerated electron and hole. ${ }^{23,26}$ To obtain information about the lifetime and mobility of the charge carriers in each QD film, a time-resolved photoluminescence (TRPL) study was carried out alongside measurements of the space-charge-limited current (SCLC). The diffusion length was then calculated from these results, which can be expressed as a function of the mobility and lifetime, $L_{\mathrm{D}}=\sqrt{D \tau}$, where $D=$ $(k T / q) \mu\left(L_{\mathrm{D}}\right.$, diffusion length; $D$, diffusion coefficient; $\tau$, carrier lifetime in the absence of a quencher; $\mu$, mobility). ${ }^{27-29}$ As shown in Figure 2a, the exciton lifetime of each TBAI- and EDT-treated QD film was extracted by fitting the TRPL decay curves with a biexponential decay function. For the TBAItreated QD films, this was characterized by two decay times: $\tau_{1}$ $=8.02 \pm 0.13 \mathrm{~ns}$ and $\tau_{2}=57.64 \pm 0.38 \mathrm{~ns}$ with prefactors $A_{1}$ of 1.37 and $A_{2}$ of 0.54 , respectively; however, for the EDT-treated QD film, the decay times were found to be $\tau_{1}=8.99 \pm 0.09 \mathrm{~ns}$ and $\tau_{2}=54.86 \pm 0.49 \mathrm{~ns}$ with prefactors $A_{1}$ of 1.69 and $A_{2}$ of 0.36 , respectively. ${ }^{28}$ In both cases, the relatively short lifetime of 
Table 1. Average Solar Cell Parameters of the TBAI-EDT QDSCs with Respect to the Different Number of Layers in the TBAIto EDT-Treated QDs ${ }^{a}$

\begin{tabular}{|c|c|c|c|c|c|c|c|}
\hline device category & $\begin{array}{c}\text { number of } \\
\text { layers } \\
\text { TBAI:EDT }\end{array}$ & $V_{\mathrm{oc}}(\mathrm{V})$ & $J_{\mathrm{sc}}\left(\mathrm{mA} \mathrm{cm} \mathrm{cm}^{-2}\right)$ & $R_{\mathrm{s}}\left(\Omega \mathrm{cm}^{2}\right)$ & $R_{\mathrm{sh}}\left(\Omega \mathrm{cm}^{2}\right)$ & FF & PCE (\%) \\
\hline \multirow[t]{2}{*}{ Category 2 (charge-balanced) } & $6: 6$ & $0.54 \pm 0.01$ & $22.38 \pm 1.47(20.53)$ & $2.11 \pm 0.79$ & $368.54 \pm 39.83$ & $0.70 \pm 0.02$ & $8.36 \pm 0.38$ \\
\hline & $4: 8$ & $0.54 \pm 0.01$ & $21.36 \pm 1.83(18.66)$ & $2.51 \pm 1.14$ & $341.99 \pm 33.82$ & $0.66 \pm 0.01$ & $7.57 \pm 0.60$ \\
\hline$\uparrow$ & $8: 4$ & $0.54 \pm 0.01$ & $22.10 \pm 1.75(19.06)$ & $7.70 \pm 1.81$ & $252.61 \pm 16.37$ & $0.55 \pm 0.01$ & $6.68 \pm 0.16$ \\
\hline Category 1 (charge-unbalanced) & $10: 2$ & $0.53 \pm 0.01$ & $21.21 \pm 1.50(18.09)$ & $8.82 \pm 0.56$ & $205.43 \pm 12.73$ & $0.51 \pm 0.02$ & $5.68 \pm 0.26$ \\
\hline
\end{tabular}

${ }^{a} J_{\text {sc }}$ extracted from the EQE spectra are quoted in the parentheses.

(a)

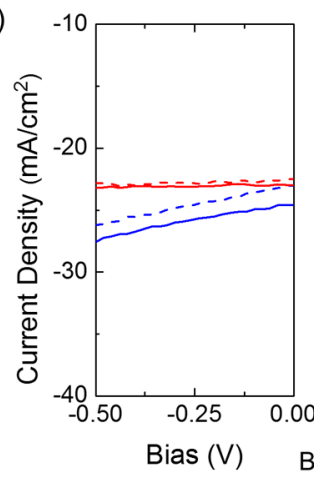

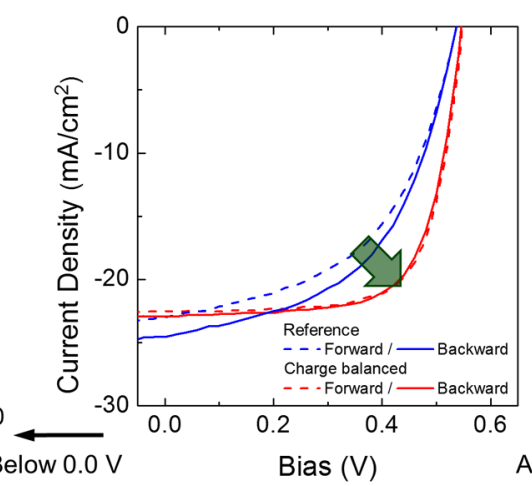
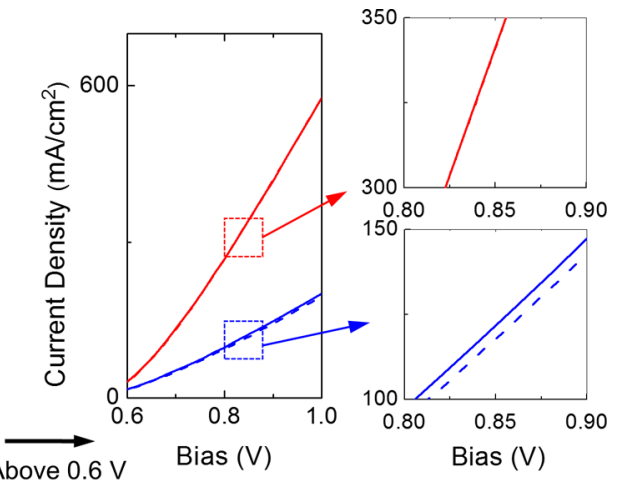

(b)

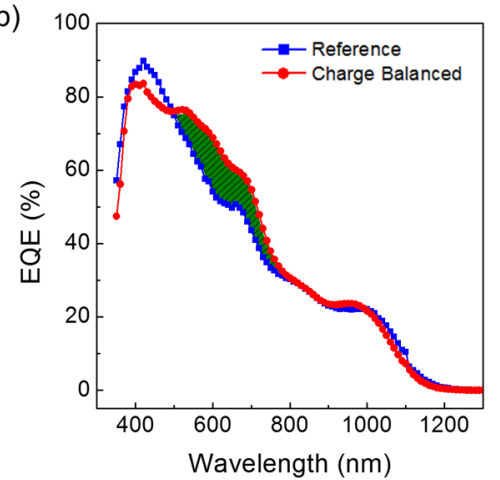

(c)

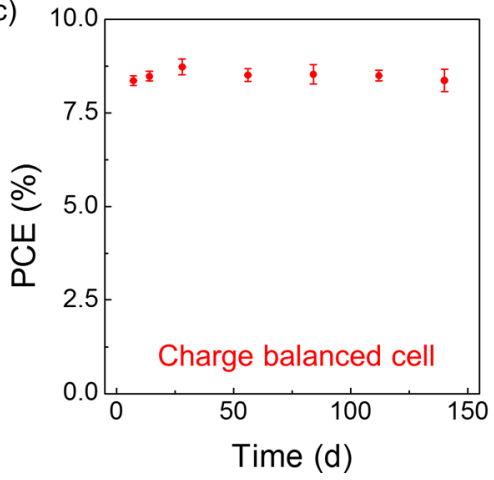

Figure 3. (a) $J-V$ characteristics of the reference (blue) and the charge-balanced cell (red) with respect to the bias voltage range ( $-0.5-0,0-$ 0.6 , and $0.6-1 \mathrm{~V}$ ), where the dotted lines correspond to a forward sweep of the bias voltage and the solid lines correspond to a reverse sweep in the bias voltage. (b) EQE curves for the reference cell and for the charge-balanced cell. (c) PCEs of the charge-balanced cells stored in ambient air, which was measured periodically over a period of almost 150 days.

the first exponent $\left(\tau_{1}\right)$ is due to a high rate of transition of interparticle charge and energy transfer, which causes rapid dissociation of the excitons, resulting in suppression of the bandgap emission, whereas the longer decay time $\left(\tau_{2}\right)$ is attributed to carrier recombination. ${ }^{29}$ The results of the TRPL measurements suggest that the carrier lifetimes in both films do not differ significantly in terms of time scales. ${ }^{30}$

The $J-V$ curves shown in Figure $2 \mathrm{~b}$ that were obtained from SCLC measurement were fitted using the Mott-Gurney law, as described in the Methods section. ${ }^{31,32}$ The mobility of each QD film was extracted from the SCLC region (slope $=2) .{ }^{33,34}$ The electron mobility of the TBAI-treated QD film (n-type) and the hole mobility of the EDT-treated QD film (p-type) were found to be $\mu_{\mathrm{e}}=3.5 \times 10^{-2} \mathrm{~cm}^{2} \mathrm{~V}^{-1} \mathrm{~s}^{-1}$ and $\mu_{\mathrm{h}}=4.4 \times 10^{-2} \mathrm{~cm}^{2} \mathrm{~V}^{-1}$ $\mathrm{s}^{-1}$, respectively. As a result, it was found that the diffusion lengths for the TBAI- and EDT-treated QD layers were comparable, as shown in Figure $2 \mathrm{c}\left(L_{\mathrm{D}}=72\right.$ and $79 \mathrm{~nm}$ for the TBAI- and EDT-treated QD films, respectively), which indicates that the distances over which the photogenerated charges are transported in both the n-type and p-type QD layers are equivalent over the same period of time. ${ }^{35,36}$

Lastly, the conductivity in both dark and light conditions was measured in order to investigate the charge transport and collection on each film, which gives information about the product of the mobility and lifetime of the carriers in a film because the conductivity is a function of mobility and steadystate charge density. Consistent with the mobility measurements, the EDT-treated QD film generated slightly higher current than that of the TBAI-treated film, in both dark and light illumination conditions, as shown in Figure $2 \mathrm{~d}$.

The results (UPS measurement in Figures 1 and 2) indicate that the formation of the QD $\mathrm{p}-\mathrm{n}$ junction by depositing $\mathrm{p}$ type $\mathrm{QD}$ layers onto $\mathrm{n}$-type $\mathrm{QD}$ layers have two significant effects on the device performance. First, the energy barrier formed by energy band bending prevents any backflow of electrons. ${ }^{9}$ Second, the exact nature of the structure of the $p-n$ 
(a)

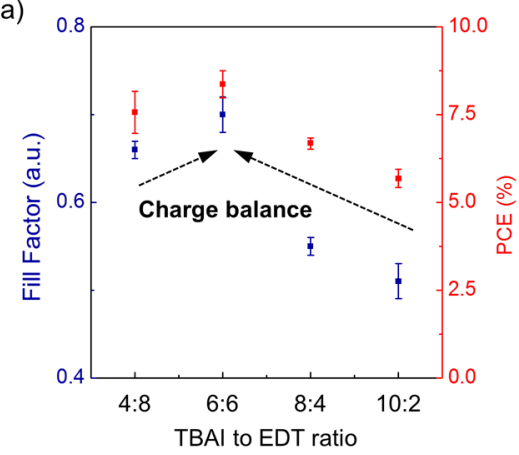

(c)

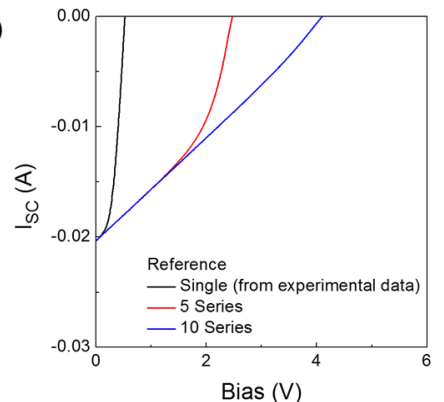

(b)

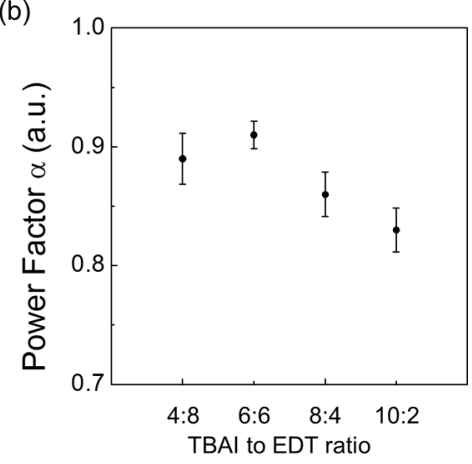

(d)

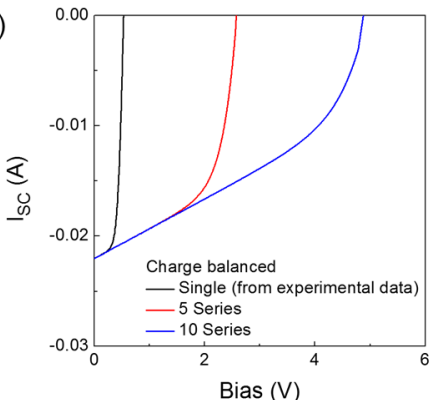

Figure 4. (a) Changes in the solar cell FF and performance conversion efficiency with respect to the different number of TBAI- and EDTtreated QD layers in the QDSCs. (b) Light intensity-dependent short-circuit current measurement of QDSCs. Simulation results for the (c) reference and (d) charge-balanced cells connected in series for a solar module application.

junction is a determinant factor in terms of efficient transport and collection of charge carriers. Taking into account these two properties of the QD junction, it was hypothesized that a judicious structural modification of the QD $\mathrm{p}-\mathrm{n}$ junction to balance charge carrier transport would further enhance the performance of a QDSC as the balanced charge system would suppress the formation of space charges and thus promote efficient transport of the charges. ${ }^{20,21,23,24}$

On the basis of the results obtained for the diffusion lengths, which show that the charge carriers in both QD films are similar to one another, we have fabricated two different categories of QDSCs with different junction structures: a reference cell (category 1), consisting of thicker n-type layers with thin p-type treated QD layers, which exhibit an imbalance in the charges in the solar cell system, and a solar cell (category 2) whereby the charges are balanced, consisting of n- and ptype layers with similar thicknesses (hereafter referred to as a charge-balanced cell). More information about the QDSCs is provided in Table 1. To present comparative behavior of the QDSCs, illustrative data is presented in Figure 3 for a reference cell with a ratio of 8:4 TBAI- to EDT-treated QD layers and for a charge-balanced cell with a layer ratio of 6:6 TBAI- to EDTtreated QD layers. As shown in Figure 3a, a prominent hysteresis and lower FF (57\%) were observed for the reference cell. In contrast, negligible hysteresis and a higher FF (72\%) were obtained for the charge-balanced cell (the measured solar cell parameters are shown in Table S2), which is attributed to efficient transport of the charge carriers due to suppression of the formation of the space charges. In addition, hysteresis in the device characteristics in the reference cell was observed throughout the full $J-V$ scanning range $(-0.5-1 \mathrm{~V})$, whereas a charge-balanced cell exhibited negligible hysteresis over the same voltage range. Considering that the contact electrodes for both types of QDSCs (ITO/ZnO/TBAI/EDT/Au) were identical, the results indicate that the hysteresis observed in the reference cell resulted from the formation of space charges not by other external causes, such as charge injection from the electrodes. These collective results indicate that balancing charges by adjusting the structure of the QD $\mathrm{p}-\mathrm{n}$ junction is necessary to realize hysteresis-free and high FF QDSCs. $^{20,21,23,24}$

Figure $3 \mathrm{~b}$ presents the external quantum efficiency (EQE) of both the reference and charge-balanced QDSCs. By integrating over the EQE curves, it was revealed that the $J_{\mathrm{sc}}$ values of the QDSCs were found to be 19.06 and $20.53 \mathrm{~mA} \mathrm{~cm}^{-2}$ for the reference and charge-balanced cell, respectively, which shows that the $J_{\mathrm{sc}}$ has been increased by $1.5 \mathrm{~mA} \mathrm{~cm}{ }^{-2}$ due to the balancing of charges. The improvement in the EQE at longer wavelengths in the charge-balanced cell (shaded in green) is related to the change in the behavior of photogenerated charge transport. As long-wavelength photons are absorbed deeper in the film due to their deeper penetration depths, the EQE result indicates that charges generated by long-wavelength photons were more efficiently transported and collected at the electrodes despite their greater distance of travel, which is attributed to the absence of space charges in the chargebalanced cell. ${ }^{9}$ Additionally, the large $J_{\mathrm{sc}}$ mismatch that was observed in the reference cell between the $J-V$ and the EQE measurements suggests that the magnitude of the hysteresis in the reference cell is detrimental and limits accurate measurement of the photocurrent using a $J-V$ sweep method. In contrast, only a slight discrepancy in $J_{\mathrm{sc}}$ was noted between the values obtained from the EQE curves when compared to the $J-$ $V$ sweep measurements for the charge-balanced cell. This is believed to be the result of balanced transport of charge carriers in a junction-structured QDSC. ${ }^{37-39}$ However, it is worth noting that, in both cases, a slight mismatch in the measured value of $J_{\mathrm{sc}}$ using a $J-V$ sweep and EQE measurement could be associated with a change in the photoactive materials under light illumination and/or measurement error. ${ }^{12}$ Lastly, the 
charge-balanced cells exhibited stable performances when they were stored in ambient air without the need for any encapsulation layer for up to 150 days, as shown in Figure $3 c$. The result indicates that the insulating $\mathrm{OA}$ ligands were successfully removed from the QD surfaces and that the QDs were sufficiently passivated by both ligands, which protects the QD layers from exposure to oxygen. ${ }^{40}$

The results revealed that the configuration of the $Q D p-n$ junction plays a significant role in balancing charges in the inverted QDSC structure. Charge balance facilitates the suppression of space charge buildup as well as efficient transport and collection of charge carriers, which results in a significantly improved FF and a concomitant enhancement in the PCE. It is worth noting that a previous study on the TBAI and EDT junction focused on the role of the EDT as an electron blocking layer (EBL). ${ }^{9}$ We further expanded the role of the EDT as a dominant hole transport layer to accomplish charge balance in the QD $\mathrm{p}-\mathrm{n}$ junction, in addition to its role as an EBL.

Further details about the effect of charge balancing in the QDSC system are illustrated in Figure 4a, demonstrating that the FF was continuously improved as the thickness of the EDTtreated QD layer became thicker until the charge balance in the QDSC approached the optimum conditions (6 layers for each of the TBAI- and EDT-treated QDs as the diffusion lengths of charge carriers are similar to one another), while the total thickness of the QD layers was maintained at approximately $300 \mathrm{~nm}$. A significant enhancement in FF $(\Delta 27 \%$, from 55 to $70 \%$ on average) led to a concomitant improvement in the solar cell performance. Further details of the QDSCs with a different number of TBAI- and EDT-treated QD layers are shown in Table 1. The values were averaged across 30 different QDSC devices. It is worth commenting that the similar $J_{\text {sc }}$ levels (slight improvement) observed for the reference and charge-balanced cells are considered to be due to the total thickness of the device being fixed at approximately $300 \mathrm{~nm}$, which is in good agreement with previous reports on charge balancing in perovskite-based solar cells. ${ }^{23,24}$

The charge-balance scheme proposed here also appeared to contribute to the suppression of nonradiative recombination due to the effective transport of the photogenerated charges, which is another indication that the FF has been enhanced. $^{20,21,23,24}$ By plotting the photocurrent of the QDSCs as a function of the light intensity on a doublelogarithmic scale, it was possible to fit the data with a linear function (see Figure S3). The extracted power factor $\alpha\left(I_{\mathrm{sc}} \propto\right.$ $I^{\alpha}$, where $I_{\mathrm{sc}}$ is the short-circuit current and $I$ is the light intensity) provides information about the charge recombination as a loss mechanism for the photocurrent. A power factor, $\alpha$, close to unity indicates that the photocurrent is determined by the generation rate of the electron-hole pairs under light illumination, whereas a value that deviates from unity suggests that the photocurrent is further limited by the formation of space charge regions as a result of the mismatch in the transport of the photogenerated charges. ${ }^{41,42}$ For the QDSCs with an equal number of $\mathrm{n}$-type and $\mathrm{p}$-type $\mathrm{QD}$ layers, the power factor, $\alpha$, was found to be close to unity as the charge carriers were balanced across the junction, as shown in Figure $4 \mathrm{~b}$. The different values of $\alpha$ for devices with a different ratio of n-type to p-type layers are shown in Table S1.

To further support that balancing charges in a QD junction is an important approach in the development of high-performance QDSC devices, we have applied this design criterion to other types of halide ligands, in particular, $\mathrm{Br}$ (tetrabutylammonium bromide (TBAB)) and $\mathrm{Cl}$ (tetrabutylammonium chloride (TBAC)). As shown in Figures S4 and S5, as well as Tables S2 and S3, a significant increase in the FF and a measurable enhancement in the PCE were observed for devices consisting of other halide ligands with an equal number of layers.

Finally, the proposed charge-balance scheme was revealed to be of prime importance in a solar module application for the practical application of QDSCs. Studies on a solar module application were performed using the MATLAB/Simulink software by comparing reference cells arranged in a series connection with that of charge-balanced cells arranged in the same configuration. More details about the simulation and an equivalent circuit diagram for the solar module are provided in the Supporting Information and Figure S6. As the number of cells connected in series was increased, a more prominent effect of the charge-balance scheme in a QDSC was observed, as shown in Figure $4 \mathrm{c}$,d. As a result of the higher $R_{\mathrm{s}}$ value that arises from space charges in the reference cells, an undesirable solar panel performance was observed when reference cells were connected in series. For example, it was found that a 29 and $61 \%$ decrease in the PCE was observed for modules with 5 and 10 reference cells connected in series, respectively. On the contrary, there was a much smaller decrease in the PCE (24 and $47 \%$ for 5 and 10 cells connected in series, respectively) in charge-balanced cells, which was attributed to the lower $R_{\mathrm{s}}$ and higher $R_{\text {sh }}$. Finally, a solar panel that consisted of 50 cells in series and 25 cells in parallel, as an example, showed a huge contrast in terms of power generation from the solar panels, which was $9.2 \mathrm{~W}$ for the panel using charge-balanced cells as oppose to $6.8 \mathrm{~W}$ for the solar panel that employed the reference cells, as shown in Figure S7. These results further demonstrate the importance of a charge-balance scheme and its potential for solar module applications using $\mathrm{PbS}$ QDs.

In summary, we have considered the fundamental role of the architecture of the QD $\mathrm{p}-\mathrm{n}$ junction in the inverted QDSC structure $\left(\mathrm{ZnO}\left(\mathrm{n}^{+}\right) / \mathrm{QD}(\mathrm{n}) / \mathrm{QD}(\mathrm{p})\right)$, which is the most frequently studied device configuration adopted in $\mathrm{PbS}$ QDSCs. By measuring the carrier lifetime and mobility of the TBAI- and EDT-treated QD films, the diffusion lengths of the corresponding films were found to be comparable to one another. On the basis of the photoelectric characteristics obtained from both QD films, we have devised a $\mathrm{p}-\mathrm{n}$ junction arrangement in order to balance charge carrier transport. A balanced QDSC exhibited negligible hysteresis, a significantly enhanced FF (>70\%), and concomitant PCE (>9\%) improvement, which are attributed to the suppression of space charges as well as efficient transport and collection of the photogenerated charges. In contrast, charge imbalance in the system leads to the formation of space charges, which resulted in a notable hysteresis in the $J-V$ curves, a low FF, and corresponding poor solar cell performance. Furthermore, a charge-balance scheme was applied to other types of halide ligands, notably $\mathrm{Br}$ and $\mathrm{Cl}$, which also showed a significantly improved FF and PCE compared to QDSCs where the charge carrier transport was not balanced. Lastly, the importance of the charge-balance strategy was further supported in simulations of a solar module application for future PbS QDSC technology.

\section{METHODS}

Solar Cell Fabrication Method. Colloidal PbS QDs and $\mathrm{ZnO}$ nanoparticles in the solution phase were synthesized according 
to a method that we have described elsewhere. ${ }^{6,8}$ A solution of $\mathrm{ZnO}$ nanoparticles in chloroform was spin-coated onto patterned ITO (thickness $150 \mathrm{~nm}$ ) glass substrates (thickness $1.1 \mathrm{~mm}$ ) at $3000 \mathrm{rpm}$ for $30 \mathrm{~s}$. The thickness of the $\mathrm{ZnO}$ layer was approximately $50 \mathrm{~nm}$. Subsequently, one drop of PbS QDs solution was spin-coated at $3000 \mathrm{rpm}$. For the solid-state ligand exchange, a tetrabutylammonium iodide (TBAI) solution in methanol with a concentration of $10 \mathrm{mg} \mathrm{mL}^{-1}$ was loaded on a $\mathrm{PbS} \mathrm{QD}$ layer and held for $30 \mathrm{~s}$, which was followed by a washing process using methanol that was carried out twice. For halide $\mathrm{Br}$ and $\mathrm{Cl}$ treatment, $10 \mathrm{mg} \mathrm{mL}^{-1}$ of tetrabutylammonium bromide (TBAB) and $20 \mathrm{mg} \mathrm{mL}^{-1}$ of tetrabutylammonium chloride (TBAC) in methanol were used, which was followed by the same washing process. All other conditions were the same unless specified otherwise. 1,2-Ethanedithiol (EDT, $0.02 \mathrm{vol} \%$ in acetonitrile) was treated by using a direct coating method without a loading time, followed by a washing process using acetonitrile, which was performed twice. The thickness of each TBAI- and EDT-treated QD layer was controlled by the number of layers deposited, and the thickness was fixed at approximately $300 \mathrm{~nm}$ for all devices where the thicknesses of a single layer of either the TBAI- or EDT-treated QD film were almost identical at 24 and $25 \mathrm{~nm}$, respectively. Gold electrodes with $100 \mathrm{~nm}$ thickness were deposited using thermal evaporation under high vacuum.

Solar Cell Characterization Method. Solar cell measurements were performed using a source meter (Keithley 4200-SCS) and a solar simulator (LOT-Quantum Design) with an AM $1.5 \mathrm{G}$ filter $\left(100 \mathrm{~mW} \mathrm{~cm}^{-2}\right)$. A standard cell (Rera system) was measured before the solar cell measurements, and all measurements were carried out in ambient air. The size of the active area was $0.03 \pm 0.002 \mathrm{~cm}^{2}$. For light intensitydependent measurements, a neutral-density (ND) filter was used to control the light intensity incident on the devices.

SCLC Devices. Electron- and hole-only devices were fabricated using the same method as the QDSCs. The structure of the electron-only device was ITO/ZnO/QD-TBAI/ZnO/Al, whereas the structure of the hole-only device was ITO/ PEDOT:PSS/TFB/QD-EDT/TFB/Au, where TFB is an abbreviation of the compound poly(9,9-dioctylfluorene-alt- $N$ (4-s-butylphenyl)-diphenylamine). Measured $I-V$ curves were fitted using the Mott-Gurney law, $J_{\mathrm{D}}=\frac{9 \varepsilon \varepsilon_{0} \mu V_{\mathrm{b}}^{2}}{8 D^{3}}$, where $V_{\mathrm{b}}$ is the applied voltage and $D$ the total thickness of the device, as described in refs 32 and 35. For the calculation of the mobility, we used a value of 21.2 for the dielectric constant of the PbS QDs, as reported in ref 14 . The total thicknesses of the electron-only device and the hole-only device were 400 and 375 $\mathrm{nm}$, respectively.

\section{ASSOCIATED CONTENT}

\section{S Supporting Information}

The Supporting Information is available free of charge on the ACS Publications website at DOI: 10.1021/acsenergylett.8b00130.

Structure of a QDSC, FTIR measurement, light intensity-dependent solar cell performance, performances of QDSCs with different types of halide ligands, and MATLAB/Simulink simulation results (PDF)

\section{AUTHOR INFORMATION}

\section{Corresponding Authors}

*E-mail: junginn.sohn@dongguk.edu (J.I.S.).

*E-mail: seungnam.cha@eng.ox.ac.uk (S.C.).

ORCID

Yuljae Cho: 0000-0003-2976-0604

Bo Hou: 0000-0001-9918-8223

Sangyeon Pak: 0000-0003-1765-3043

John Hong: 0000-0002-1513-8622

Young-Woo Lee: 0000-0003-0777-8221

Jae Eun Jang: 0000-0002-8523-1785

Henry J. Snaith: 0000-0001-8511-790X

Jung Inn Sohn: 0000-0002-3155-4327

SeungNam Cha: 0000-0001-6284-8312

Author Contributions

${ }^{\nabla}$ Y.C. and B.H. contributed equally to this work.

Notes

The authors declare no competing financial interest.

\section{ACKNOWLEDGMENTS}

This work was supported by the National Research Foundation of Korea (NRF) (2015M2A2A6A02045252) and the European Research Council under ERC Grant Agreement Number 340538 and the European Commission Horizon 2020 under Grant Agreement Number 685758.

\section{REFERENCES}

(1) Lewis, N. S. Toward Cost-Effective Solar Energy Use. Science 2007, 315, 798-801.

(2) Sargent, E. H. Colloidal Quantum Dot Solar Cells. Nat. Photonics 2012, 6, 133-135.

(3) Service, R. F. Solar Energy. Can the Upstarts Top Silicon? Science 2008, 319, 718-720.

(4) Kim, J. Y.; Voznyy, O.; Zhitomirsky, D.; Sargent, E. H. 25th Anniversary Article: Colloidal Quantum Dot Materials and Devices: A Quarter-Century of Advances. Adv. Mater. 2013, 25, 4986-5010.

(5) Cao, Y.; Stavrinadis, A.; Lasanta, T.; So, D.; Konstantatos, G. The Role of Surface Passivation for Efficient and Photostable $\mathrm{PbS}$ Quantum Dot Solar Cells. Nat. Energy 2016, 1, 16035.

(6) Hou, B.; Cho, Y.; Kim, B. S.; Hong, J.; Park, J. B.; Ahn, S. J.; Sohn, J. I.; Cha, S.; Kim, J. M. Highly Monodispersed PbS Quantum Dots for Outstanding Cascaded-Junction Solar Cells. ACS Energy Lett. 2016, 1, 834-839.

(7) Michalet, X.; Pinaud, F. F.; Bentolila, L. A.; Tsay, J. M.; Doose, S.; Li, J. J.; Sundaresan, G.; Wu, A. M.; Gambhir, S. S.; Weiss, S. Quantum Dots for Live Cells, in Vivo Imaging, and Diagnostics. Science 2005, 307, 538-544.

(8) Hou, B.; Cho, Y.; Kim, B. - S.; Ahn, D.; Lee, S.; Park, J. B.; Lee, Y.; Hong, J.; Im, H.; Morris, S. M.; et al. Red Green Blue Emissive Lead Sulfide Quantum Dots: Heterogeneous Synthesis and Applications. J. Mater. Chem. C 2017, 5, 3692-3698.

(9) Chuang, C. M.; Brown, P. R.; Bulović, V.; Bawendi, M. G. Improved Performance and Stability in Quantum Dot Solar Cells through Band Alignment Engineering. Nat. Mater. 2014, 13, 796-801.

(10) Chuang, C. M.; Maurano, A.; Brandt, R. E.; Hwang, G. W.; Jean, J.; Buonassisi, T.; Bulović, V.; Bawendi, M. G. Open-Circuit Voltage Deficit, Radiative Sub-Bandgap States, and Prospects in Quantum Dot Solar Cells. Nano Lett. 2015, 15, 3286-3294.

(11) Liu, M.; de Arquer, F.; Li, Y.; Lan, X.; Kim, G.; Voznyy, O.; Jagadamma, L. K.; Abbas, A. S.; Hoogland, S.; Lu, Z.; et al. DoubleSided Junctions Enable High-Performance Colloidal-Quantum-Dot Photovoltaics. Adv. Mater. 2016, 28, 4142-4148.

(12) Lan, X.; Voznyy, O.; Kiani, A.; García de Arquer, F. P.; Abbas, A. S.; Kim, G.; Liu, M.; Yang, Z.; Walters, G.; Xu, J.; et al. Passivation 
Using Molecular Halides Increases Quantum Dot Solar Cell Performance. Adv. Mater. 2016, 28, 299-304.

(13) Liu, M.; Voznyy, O.; Sabatini, R.; García de Arquer, F. P.; Munir, R.; Balawi, A. H.; Lan, X.; Fan, F.; Walters, G.; Kirmani, A. R.; et al. Hybrid Organic-Inorganic Inks Flatten the Energy Landscape in Colloidal Quantum Dot Solids. Nat. Mater. 2017, 16, 258-263.

(14) Aqoma, H.; Al Mubarok, M.; Hadmojo, W. T.; Lee, E.; Kim, T.; Ahn, T. K.; Oh, S.; Jang, S. High-Efficiency Photovoltaic Devices Using Trap-Controlled Quantum-Dot Ink prepared via Phase-Transfer Exchange. Adv. Mater. 2017, 29, 1605756.

(15) Hong, J.; Hou, B.; Lim, J.; Pak, S.; Kim, B. - S.; Cho, Y.; Lee, J.; Lee, Y.; Giraud, P.; Lee, S.; et al. Enhanced Charge Carrier Transport Properties in Colloidal Quantum Dot Solar Cells via Organic and Inorganic Hybrid Surface Passivation. J. Mater. Chem. A 2016, 4, 18769-18775.

(16) Rekemeyer, P. H.; Chang, S.; Chuang, C. M.; Hwang, G. W.; Bawendi, M. G.; Gradečak, S. Enhanced Photocurrent in PbS Quantum Dot Photovoltaics via $\mathrm{ZnO}$ Nanowires and Band Alignment Engineering. Adv. Energy Mater. 2016, 6, 1600848.

(17) Azmi, R.; Oh, S.; Jang, S. High-Efficiency Colloidal Quantum Dot Photovoltaic Devices Using Chemically Modified Heterojunctions. ACS Energy Lett. 2016, 1, 100-106.

(18) Tang, J.; Liu, H.; Zhitomirsky, D.; Hoogland, S.; Wang, X.; Furukawa, M.; Levina, L.; Sargent, E. H. Quantum Junction Solar Cells. Nano Lett. 2012, 12, 4889-4894.

(19) Rekemeyer, P.; Chuang, C. M.; Bawendi, M. G.; Gradecak, S. Minority Carrier Transport in Lead Sulfide Quantum Dot Photovoltaics. Nano Lett. 2017, 17, 6221-6227.

(20) Chiang, C.; Wu, C. Bulk Heterojunction Perovskite-PCBM Solar Cells with High Fill Factor. Nat. Photonics 2016, 10, 196-200.

(21) Zhou, H.; Chen, Q.; Li, G.; Luo, S.; Song, T. B.; Duan, H. S.; Hong, Z.; You, J.; Liu, Y.; Yang, Y. Interface Engineering of Highly Efficient Perovskite Solar Cells. Science 2014, 345, 542-546.

(22) Brown, P. R.; Kim, D.; Lunt, R. R.; Zhao, N.; Bawendi, M. G.; Grossman, J. C.; Bulović, V. Energy Level Modification in Lead Sulfide Quantum Dot Thin Films through Ligand Exchange. ACS Nano 2014, 8,5863 .

(23) Chen, K.; Hu, Q.; Liu, T.; Zhao, L.; Luo, D.; Wu, J.; Zhang, Y.; Zhang, W.; Liu, F.; Russell, T. P.; et al. Charge-Carrier Balance for Highly Efficient Inverted Planar Heterojunction Perovskite Solar Cells. Adv. Mater. 2016, 28, 10718-10724.

(24) Heo, J. H.; Han, H. J.; Kim, D.; Ahn, T. K.; Im, S. H. HysteresisLess Inverted $\mathrm{CH}_{3} \mathrm{NH}_{3} \mathrm{PbI}_{3}$ Planar Perovskite Hybrid Solar Cells with 18.1\% Power Conversion Efficiency. Energy Environ. Sci. 2015, 8, $1602-1608$.

(25) Ponseca, C. S., Jr; Savenije, T. J.; Abdellah, M.; Zheng, K.; Yartsev, A.; Pascher, T.; Harlang, T.; Chabera, P.; Pullerits, T.; Stepanov, A.; et al. Organometal Halide Perovskite Solar Cell Materials Rationalized: Ultrafast Charge Generation, High and Microsecond-Long Balanced Mobilities, and Slow Recombination. J. Am. Chem. Soc. 2014, 136, 5189-5192.

(26) Xing, G.; Mathews, N.; Sun, S.; Lim, S. S.; Lam, Y. M.; Grätzel, M.; Mhaisalkar, S.; Sum, T. C. Long-Range Balanced Electron- and Hole-Transport Lengths in Organic-Inorganic $\mathrm{CH}_{3} \mathrm{NH}_{3} \mathrm{PbI}_{3}$. Science 2013, 342, 344-347.

(27) Parrott, E. S.; Milot, R. L.; Stergiopoulos, T.; Snaith, H. J.; Johnston, M. B.; Herz, L. M. Effect of Structural Phase Transition on Charge-Carrier Lifetimes and Defects in $\mathrm{CH}_{3} \mathrm{NH}_{3} \mathrm{SnI}_{3}$ Perovskite. J. Phys. Chem. Lett. 2016, 7, 1321-1326.

(28) Sanchez, R. S.; de la Fuente, M. S.; Suarez, I.; Muñoz-Matutano, G.; Martinez-Pastor, J. P.; Mora-Sero, I. Tunable Light Emission by Exciplex State Formation between Hybrid Halide Perovskite and Core/Shell Quantum Dots: Implications in Advanced LEDs and Photovoltaics. Sci. Adv. 2016, 2, e1501104.

(29) Moroz, P.; Kholmicheva, N.; Mellott, B.; Liyanage, G.; Rijal, U.; Bastola, E.; Huband, K.; Khon, E.; McBride, K.; Zamkov, M. Suppressed Carrier Scattering in CdS-Encapsulated PbS Nanocrystal Films. ACS Nano 2013, 7, 6964-6977.
(30) Guglietta, G. W.; Diroll, B. T.; Gaulding, E. A.; Fordham, J. L.; Li, S.; Murray, C. B.; Baxter, J. B. Lifetime, Mobility, and Diffusion of Photoexcited Carriers in Ligand-Exchanged Lead Selenide Nanocrystal Films Measured by Time-Resolved Terahertz Spectroscopy. ACS Nano 2015, 9, 1820-1828.

(31) Dong, Q.; Fang, Y.; Shao, Y.; Mulligan, P.; Qiu, J.; Cao, L.; Huang, J. Electron-Hole Diffusion Lengths $>175 \mu \mathrm{m}$ in SolutionGrown $\mathrm{CH}_{3} \mathrm{NH}_{3} \mathrm{PbI}_{3}$ Single Crystals. Science 2015, 347, 967-970.

(32) Guo, X.; Zhou, N.; Lou, S. J.; Smith, J.; Tice, D. B.; Hennek, J. W.; Ortiz, R. P.; Navarrete, J. T. L.; Li, S.; Strzalka, J.; et al. Polymer Solar Cells with Enhanced Fill Factors. Nat. Photonics 2013, 7, 825833.

(33) Li, M.; Li, B.; Cao, G.; Tian, J. Monolithic MAPbI ${ }_{3}$ Films for High-Efficiency Solar Cells via Coordination and a Heat Assisted Process. J. Mater. Chem. A 2017, 5, 21313-21319.

(34) Yun, D. Y.; Park, H. M.; Kim, S. W.; Kim, S. W.; Kim, T. W. Enhancement of Memory Margins for Stable Organic Bistable Devices Based on Graphene-Oxide Layers due to Embedded CuInS 2 Quantum Dots. Carbon 2014, 75, 244-248.

(35) Zhitomirsky, D.; Voznyy, O.; Levina, L.; Hoogland, S.; Kemp, K. W.; Ip, A. H.; Thon, S. M.; Sargent, E. H. Engineering Colloidal Quantum Dot Solids within and beyond the Mobility-Invariant Regime. Nat. Commun. 2014, 5, 3803.

(36) Zhitomirsky, D.; Furukawa, M.; Tang, J.; Stadler, P.; Hoogland, S.; Voznyy, O.; Liu, H.; Sargent, E. H. N-Type Colloidal-QuantumDot Solids for Photovoltaics. Adv. Mater. 2012, 24, 6181-6185.

(37) Yang, Z.; Janmohamed, A.; Lan, X.; García de Arquer, F. P.; Voznyy, O.; Yassitepe, E.; Kim, G.; Ning, Z.; Gong, X.; Comin, R.; et al. Colloidal Quantum Dot Photovoltaics Enhanced by Perovskite Shelling. Nano Lett. 2015, 15, 7539-7543.

(38) Pradhan, S.; Stavrinadis, A.; Gupta, S.; Christodoulou, S.; Konstantatos, G. Breaking the Open-Circuit Voltage Deficit Floor in $\mathrm{PbS}$ Quantum Dot Solar Cells through Synergistic Ligand and Architecture Engineering. ACS Energy Lett. 2017, 2, 1444-1449.

(39) Stavrinadis, A.; Pradhan, S.; Papagiorgis, P.; Itskos, G.; Konstantatos, G. Suppressing Deep Traps in PbS Colloidal Quantum Dots via Facile Iodide Substitutional Doping for Solar Cells with Efficiency $>10 \%$. ACS Energy Lett. 2017, 2, 739-744.

(40) Ning, Z.; Voznyy, O.; Pan, J.; Hoogland, S.; Adinolfi, V.; Xu, J.; Li, M.; Kirmani, A. R.; Sun, J.; Minor, J.; et al. Air-Stable n-Type Colloidal Quantum Dot Solids. Nat. Mater. 2014, 13, 822-828.

(41) Zhao, N.; Osedach, T. P.; Chang, L.; Geyer, S. M.; Wanger, D.; Binda, M. T.; Arango, A. C.; Bawendi, M. G.; Bulovic, V. Colloidal PbS Quantum Dot Solar Cells with High Fill Factor. ACS Nano 2010, 4, $3743-3752$.

(42) Mihailetchi, V.; Wildeman, J.; Blom, P. Space-Charge Limited Photocurrent. Phys. Rev. Lett. 2005, 94, 126602. 